\title{
MEIN ERSTER (UND ZWEITER) EINBLICK IN DIE ÖSTER- REICHISCHE BIBLIOTHEKSLANDSCHAFT - PRAKTIKA AN DEN BIBLIOTHEKEN DER TU WIEN UND MEDUNI WIEN
}

\author{
von Tereza Kalová
}

Zusammenfassung: Ein Bericht über Praktika an zwei großen Universitätsbibliotheken in Wien, welche die Autorin im Rahmen des Masterstudiums der Bibliotheksund Informationswissenschaft an der Humboldt-Universität zu Berlin absolviert hat. Das Praktikum an der TU Wien Bibliothek im Oktober 2018 beschäftige sich hauptsächlich mit Erwerbung und Publikationsservices. Das Praktikum an der Universitätsbibliothek der Medizinischen Universität Wien von November bis Dezember 2018 umfasste systembibliothekarische Tätigkeiten.

Schlagwörter: Praktika; Universitätsbibliothek der TU Wien; Universitätsbibliothek der Medizinischen Universität Wien; Bericht

\section{THE WORLD OF AUSTRIAN LIBRARIANSHIP: MY FIRST (AND SECOND) IMPRESSIONS - INTERNSHIPS AT THE TU WIEN AND MEDICAL UNIVERSITY OF VIENNA}

\begin{abstract}
Included in this report are accounts of two internships at major university libraries in Vienna, undertaken in the fulfillment of a Master of Arts degree in Library and Information Science from Humboldt University of Berlin. The author spent October 2018 at the TU Wien (Vienna University of Technology) Library, focusing on the acquisition department and publishing services. The second internship was completed from November to December 2018 at the Medical University of Vienna Library and covered mainly the tasks of a systems librarian.
\end{abstract}

Keywords: Internships; Vienna University of Technology library; Medical University of Vienna library; report

DOI: https://doi.org/10.31263/voebm.v72i1.2282

(c) Tereza Kalová

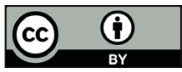

Dieses Werk ist lizenziert unter einer

Creative-Commons-Lizenz Namensnennung 4.0 International 
Im September 2018 habe ich den großen aufregenden Schritt von München, wo ich an der Bayerischen Staatsbibliothek und der Internationalen Jugendbibliothek tätig war, nach Wien gewagt. Man liest immer wieder, dass Wien die lebenswerteste Stadt der Welt ist und ich kann dies nach einem halben Jahr nur bestätigen! Aufgrund meines Wunsches in Wien Fuß zu fassen, habe ich mich entschieden, den letzten Teil meiner studienbezogenen Praktika an zwei großen wissenschaftlichen Wiener Bibliotheken zu absolvieren. Da ich von Haus aus Geisteswissenschaftlerin bin, wollte ich über eine Tätigkeit an fachfremden Universitätsbibliotheken neue Perspektiven gewinnen. Ich habe mich deshalb an der TU Wien und der Medizinischen Universität Wien beworben und mit Freude von beiden Bibliotheken Zusagen erhalten.

Am Montag, den 1. Oktober 2018 stand ich daher vor der Tür der Leiterin der TU Bibliothek, Mag. ${ }^{a}$ Beate Guba. Nach einem kurzen Gespräch habe ich einen ausführlichen Praktikumsplan bekommen, in dem auf meine Wünsche eingegangen wurde, in der Erwerbung und im Bereich Publikationsservices mitzuarbeiten. Schon die Einführung in die tägliche Arbeit in der Bibliothek hat eine große Überraschung mit sich gebracht - die TU Wien Bibliothek wird nach wie vor zweischichtig organisiert! So betreuen die Mitarbeiter*innen vor allem in der Erwerbung über 90 Etats für all die unterschiedlich großen Hand- und Institutsbibliotheken. Da ich dieses Konzept nur theoretisch aus meinem Studium kannte, fand ich die praktische Umsetzung unglaublich spannend.

Das Sammeln von Literatur und die damit verbundene Idee einer zentralen Bibliothek war schon immer ein hochpolitisches Thema an Universitäten, vor allem wenn es um Printbestände geht. Es stellt sich die Frage, was wichtiger ist: die Erreichbarkeit der häufig benutzten Bände für Professoren und Professorinnen in greifbarer Nähe auf der einen Seite oder auf der Anderen das interdisziplinäre Arbeiten und allgemeiner Zugriff für Alle an einem Ort. Die vielen Budgets und Vorstellungen einzelner Professoren unter einen Hut zu bringen ist natürlich nicht leicht. Ich habe sehr schnell gemerkt, dass die tatsächliche Erwerbung und Katalogisierung häufig an die zweite Stelle rücken. Der Erfolg wird hier nicht nur durch gewissenhafte Bestellungen bestimmt, sondern vielmehr durch serviceorientiertes Arbeiten und vor allem offene Kommunikation auf allen Ebenen!

Jeder, der im österreichischen Bibliothekswesen arbeitet, hat bestimmt von den vielen innovativen Ideen gehört, die Frau Guba an der TU einsetzt. In den letzten Monaten wurde dort ein Zentrum für Forschungsdatenmanagement, ein neuer Universitätsverlag „TU Wien Academic Press" sowie ein neues Angebot an bibliometrischen Auswertungen für Universitätsan- 
gehörige eingeführt. Diese Neuerungen haben für ein außergewöhnlich breites Spektrum an Praktikumstätigkeiten gesorgt. So hatte ich die einzigartige Möglichkeit, über das neue ORCID-Konsortium direkt von der Leiterin Paloma Marín Arraiza zu erfahren und konnte mit einem Professor für Architektur über das Layout für sein neues Buch diskutieren. Darüber hinaus habe ich die ersten Web of Science- und SCOPUS-Abfragen für eine bibliometrische Auswertung für ein gesamtes Institut durchgeführt.

Die Monate November und Dezember durfte ich dann an der UB der MedUni Wien im AKH verbringen. Wer schon mal die österreichische Hauptstadt besucht hat, der konnte die Kulisse des Allgemeinen Krankenhauses wahrscheinlich nicht übersehen. Wörter wie „einladend“ oder „freundlich" sind wahrscheinlich nicht die üblichen Bezeichnungen dafür. Nichtsdestotrotz fallen mir genau diese zwei Worte ein, um meine Eindrücke von der Bibliothek, deren Leitung und den Mitarbeiter*innen zu beschreiben.

Früherwaren fürmich, wie fürvieleandereangehende Bibliothekar*innen, systembibliothekarische Tätigkeiten vielleicht ein wenig zu weit vom traditionellen Verständnis des Berufs entfernt. Als ich aber angefangen habe, die nach dem Umstieg von Aleph auf ALMA notwendige Datenbereinigung durchzuführen, habe ich direkt gemerkt, wie spannend die Aufgabe sein kann! Man fühlt sich wie ein virtueller Detektiv, während man das Labyrinth an Datensätzen durchsucht und korrigiert, um genau den einen Richtigen für die Benützer zu finden.

Von der Adjustierung bis zur Managementebene hat sich jeder an der UB für mich und meine Fragen viel Zeit genommen und mich aktiv in meinem Praktikum unterstützt. Ich durfte eine Führung durch die historischen Bestände im Josephinum begleiten und weiter auch an dem 4. Österreichischen TeachMeet und einem Treffen des Arbeitspakets Metadaten (e-Infrastractures Austria Plus) teilnehmen. Der Leiter der Bibliothek, Mag. Bruno Bauer, hat mit mir über Open Access und Forschungsdatenmanagement in Österreich diskutiert und Mag. ${ }^{a}$ Karin Cepicka hat mir sogar wertvolle Tipps für die Jobsuche mitgegeben. Ich habe über das Praktikum sehr viele interessante Menschen aus dem Bibliothekswesen kennengelernt, u. a. Frau Dr. ${ }^{\text {in }}$ Susanne Blumesberger. Mit ihrer Unterstützung verfasse ich jetzt im Rahmen eines Projekts an der Universität Wien meine Masterarbeit zum Thema Metadaten für Forschungsdaten.

Nicht zuletzt war meine Hoffnung, über die Praktika an eine Stelle in Wien zu gelangen. Dank der ausgezeichneten Erfahrung und Betreuung seitens des Bibliotheksmanagements sowie der netten Kolleginnen und Kollegen, freue ich mich sehr seit März als Spezialistin für Forschungs- 
datenmanagement an der WU Wien zu arbeiten und die österreichische Bibliothekslandschaft von innen mitgestalten zu können!

Tereza Kalová, MA (Res) Wirtschaftsuniversität Wien, Universitätsbibliothek E-Mail: tereza.kalova@wu.ac.at 